\title{
Infliximab in Combination with Low-Dose Acitretin in Generalized Pustular Psoriasis: A Report of Two Cases and Review of the Literature
}

\section{Marta Kołt-Kamińska Magdalena Żychowska (D) Adam Reich (D)}

Department of Dermatology, Institute of Medical Sciences, Medical College of Rzeszów University, Rzeszów, Poland
Correspondence: Magdalena Żychowska Department of Dermatology, Institute of Medical Sciences, Medical College of Rzeszów University, Reszów, Poland Tel +48 66-942-2237

Fax +48 I7 8666294

Email magda.zychowska@gmail.com

\begin{abstract}
Generalized pustular psoriasis (GPP) is a severe, life-threatening disease that represents a major therapeutic challenge. There is a lack of randomized controlled trials assessing the efficacy of various treatment options for GPP. TNF $\alpha$ inhibitors have proven to be effective and are increasingly used in this indication. In the current paper, we present two patients with GPP treated with infliximab (Ifx) and a literature review appraising currently available data on the use of Ifx in GPP. Case 1 was a 73 -year-old woman with GPP who exhibited lack of treatment response or primary intolerance to standard therapeutic options (high-dose acitretin, methotrexate, cyclosporine A, and methylprednisolone). However, Ifx therapy combined with low-dose acitretin resulted in rapid and sustained resolution of skin lesions. Case 2 was a 60 -year-old man with GPP and numerous comorbidities who was initially treated with Ifx in combination with methotrexate, with good treatment response for 9 months. Following an infectioninduced flare of GPP at week 38, methotrexate was discontinued in favor of low-dose acitretin and Ifx continued. This regimen again resulted in rapid resolution of pustules. We present these cases to highlight the advantage of long-term Ifx therapy with low-dose acitretin in GPP.
\end{abstract}

Keywords: infliximab, anti-TNF $\alpha$, generalized pustular psoriasis, acitretin

\section{Introduction}

Generalized pustular psoriasis (GPP), also called von Zumbusch psoriasis, is characterized by the presence of widespread sterile pustules on erythematous skin, usually of rapid onset and accompanied by systemic symptoms, including fever, malaise, nausea, and arthralgia. ${ }^{1-4}$ Prompt management is required, as the condition may lead to life-threatening complications. ${ }^{5,6}$ Classical therapies, including acitretin, cyclosporine A (CsA), or methotrexate (Mtx), are usually used as firstline options, but their onset of action may be delayed and their application associated with significant systemic toxicity. The National Psoriasis Foundation recommends infliximab (Ifx) as the first-line biologic for severe GPP in adults. It should also be considered as a second-line therapeutic option for juvenile GPP and firstline therapy for GPP (or impetigo herpetiformis) developing during pregnancy (category B). ${ }^{5}$ However, due to the rarity of GPP, these recommendations are mainly based on case reports and expert opinions. Therefore, in the current paper, we present two patients with GPP treated with Ifx. We also review the existing 
literature on the treatment of GPP in adults with Ifx and discuss management options for pustular flares during long-term Ifx therapy.

\section{Case Reports Case I}

A 73-year-old woman with several months' history of recalcitrant GPP was admitted to the Department of Dermatology in Rzeszów due to severe exacerbation of her skin condition. Her personal and family history of psoriasis was negative. Prior treatment included acitretin (initially at $0.8 \mathrm{mg} / \mathrm{kg}$ and gradually tapered down due to side effects, including massive hair loss, epistaxis, and severe skin hypersensitivity, and eventually discontinued due to lack of efficacy at lower doses in monotherapy), CsA 300-400 mg/day (discontinued due to nephrotoxicity), oral Mtx $15 \mathrm{mg} /$ week (discontinued due to primary lack of efficacy), and oral methylprednisolone $16 \mathrm{mg}$ /day (discontinued due to lack of efficacy). On admission, she presented with widespread painful erythematous plaques with coalescing pustules (Figure 1A and B), accompanied by systemic symptoms (fever, malaise, and chills). Laboratory results showed anemia (hemoglobin $9.9 \mathrm{~g} /$ $\mathrm{dL}$, red blood-cell count $3.32 \times 10^{6} / \mathrm{uL}$ ), accelerated ESR $(28 \mathrm{~mm})$, and elevated CRP $(5.6 \mathrm{mg} / \mathrm{dL})$. Treatment with acitretin was reintroduced at $50 \mathrm{mg} /$ day $(0.8 \mathrm{mg} / \mathrm{kg})$; , though with only minimal improvement. Therefore, after 3 weeks, Ifx $(5 \mathrm{mg} / \mathrm{kg}$ intravenously) was initiated with concurrent tapering down of acitretin. Rapid improvement of the skin was observed after the first infusion of Ifx. The second dose was

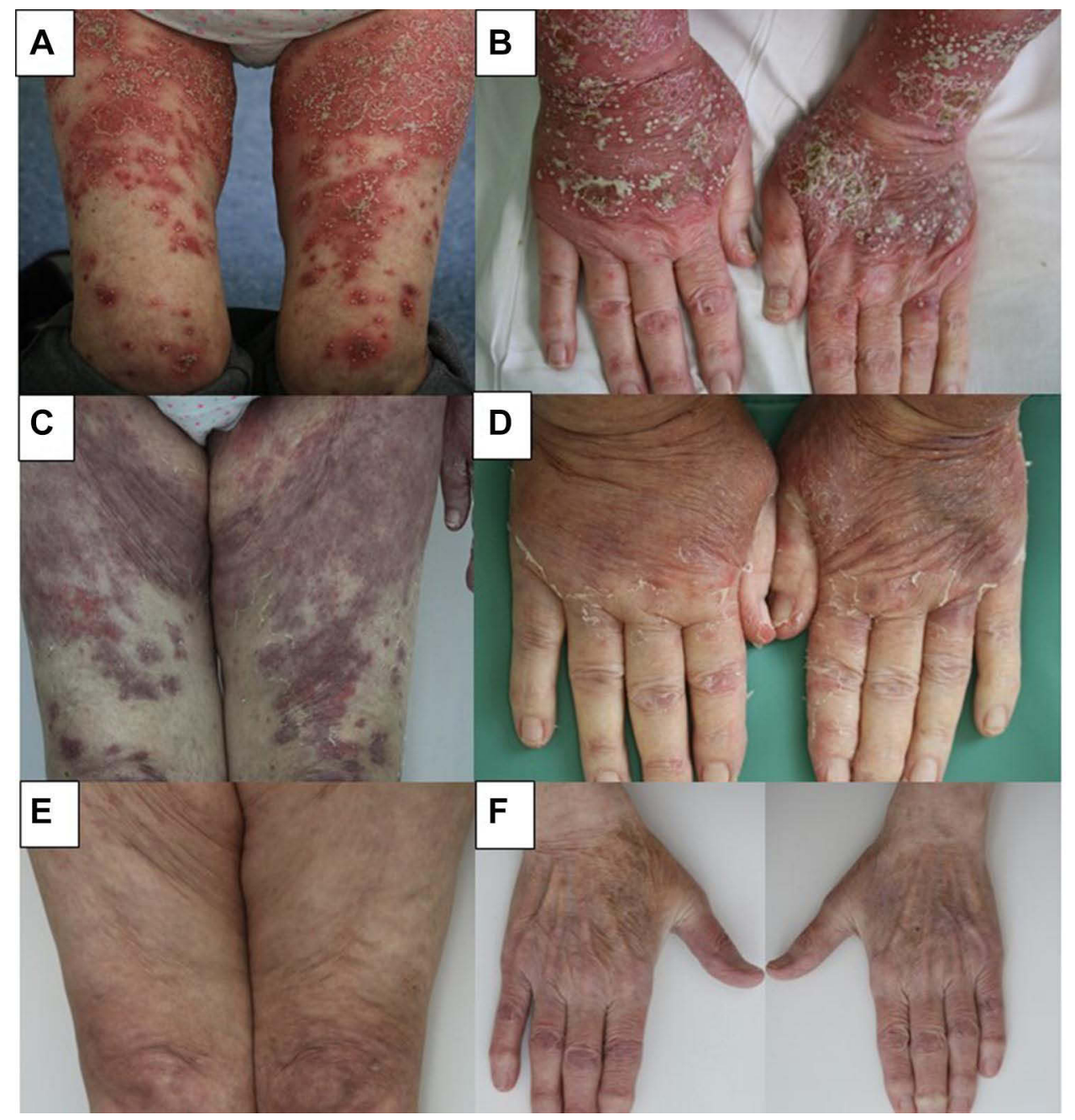

Figure I Case I (A and, B) widespread erythematous plaques and coalescing pustules on day 0; (C and, D) widespread postinflammatory hyperpigmentation with residual erythema and exfoliation on the distal parts of the upper limbs at week 2; (E and, F) almost-clear skin with discrete residual erythema on the dorsa of the hands at week 18. 
administered 2 weeks later, as per the standard dosing schedule. At this point, the patient exhibited widespread postinflammatory hyperpigmentation with residual erythema and exfoliation on the distal parts of the upper limbs (Figure 1C and D). The third infusion of Ifx, initially scheduled for week 6 , had to be postponed until week 10 due to segmental herpes zoster. The fourth infusion of Ifx was administered at week 18 . At that time, only minimal residual erythema was present on the dorsa of the hands, with complete resolution of the rest of her skin (Figure 1E and F). Acitretin was given at a slowly tapered dose until week 18 , when it was discontinued. However, the patient experienced severe exacerbation of GPP just before the fifth Ifx infusion. Ifx was administered according to schedule (week 26), and acitretin was restarted at $20 \mathrm{mg} /$ day $(0.3 \mathrm{mg} / \mathrm{kg})$, with rapid resolution of pustules. No further pustules have developed in the meantime. The patient has thus far received nine infusions of Ifx combined with acitretin (currently $20 \mathrm{mg}$ /day). No significant side effects, with the exception of herpes zoster at week 6 , have been noted during the entire period of treatment.

\section{Case 2}

A 60 -year-old man with a 40 -year history of plaque psoriasis was admitted to the Department of Dermatology in Rzeszów due to GPP triggered by upper respiratory tract infection. He had multiple comorbidities, including obesity, hypertension, thoracic aortic aneurysm, coronary artery disease, pulmonary fibrosis, fatty liver, and previous $\mathrm{HBV}$ infection. $\mathrm{He}$ had already suffered several erythrodermic exacerbations, and treatment modalities already attempted at that point included acitretin, Mtx, and psoralen and ultraviolet A. On admission, the patient presented with widespread pustules on a background of erythema with psoriatic plaques on the extensor surfaces of the elbows and knees, accompanied by arthralgia and general malaise (Figure 2A and B). Laboratory investigations revealed leukocytosis $\left(12.95 \times 10^{9}\right.$ cells $\left./ \mathrm{L}\right)$ with neutrophilia $\left(10.16 \times 10^{9} \quad\right.$ cells/L), accelerated $\quad$ ESR $(46 \mathrm{~mm})$ and elevated CRP $(19.8 \mathrm{mg} / \mathrm{dL})$. Taking into consideration the severity of the GPP and his comorbidities, therapy with Ifx $(5 \mathrm{mg} / \mathrm{kg})$ in combination with Mtx (7.5 mg/week) was initiated. Significant improvement was observed after the first Ifx infusion. Residual erythema was present on the trunk, lower legs, and forearms at week 2 (Figure 2C and D). Treatment with Ifx was continued according to the standard regimen (weeks 0,2 , and 6 and 8-weekly thereafter). At week 30 , the skin was clear, apart from residual plaques on the elbows (Figure $2 \mathrm{E}$ and F). Mtx $7.5 \mathrm{mg} /$ week was continued throughout the treatment with Ifx. No adverse effects were detected. Nine months after initiation of Ifx therapy (prior to the seventh infusion), the patient once again experienced a severe exacerbation of GPP following an upper respiratory tract infection. Mtx was discontinued, and acitretin at a dose of $35 \mathrm{mg} / \mathrm{day}(0.35 \mathrm{mg} / \mathrm{kg})$ was initiated. The seventh dose of Ifx was administered according to schedule. Again, rapid improvement of GPP was achieved. Since then, only persistent psoriatic plaques on the extensor surfaces of his elbows and knees and in the sacral region have been observed. He remains on a combination regimen of Ifx and acitretin (4 months following the last flare), without significant adverse events. No pustular flares have been observed since the introduction of acitretin.

This study was exempt from institutional review board approval. Written informed consent was obtained from both patients for publication of their photographs and this report.

\section{Discussion}

Ifx is a chimeric anti-TNF $\alpha$ monoclonal antibody that is used for the treatment of psoriasis and psoriatic arthritis. $^{6}$ As mentioned in the Introduction, Ifx is also recommended in GPP, despite the lack of reliable clinical studies. ${ }^{5}$ Therefore, we performed a comprehensive search of three medical databases (PubMed, Scopus, and Web of Science) using the search terms "infliximab" and "generalized pustular psoriasis" to identify all reported cases of GPP treated with Ifx. Reports of impetigo herpetiformis in pregnant women were not included in the analysis. Cases of GPP in adults treated with Ifx are summarized in Table 1. Data from the literature, based predominantly on single case reports and case series, highlight high efficacy and rapid onset of action of Ifx in GPP. ${ }^{1-4,6-20}$ However, we found a large variety of therapeutic strategies undertaken. Cases of singledose Ifx ${ }^{8-10,15,17,20,23}$ and longer therapy (up to 3 years) ${ }^{1-4,6-9,12-14,16-19,21}$ were among those reported. In cases of prolonged treatment, the standard dosing regimen used in plaque psoriasis (infusions of $5 \mathrm{mg} / \mathrm{kg}$ Ifx at weeks 0,2 , and 6 and subsequently every 8 weeks) 


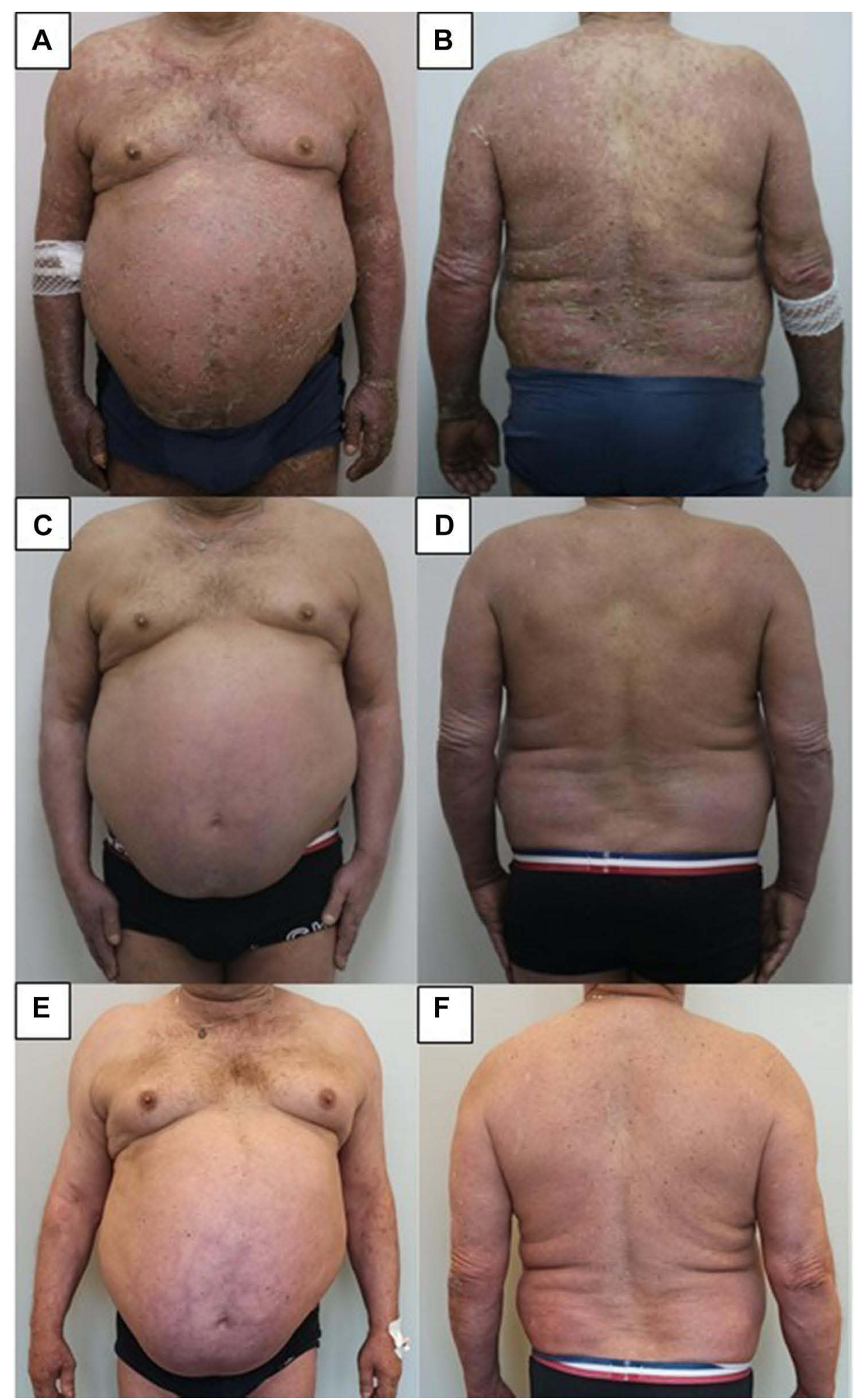

Figure 2 Case 2 (A and, B) widespread erythematous plaques with coalescing pustules on day 0; (C and, D) significant improvement with residual erythema on the trunk and upper limbs at week 2; (E and, F) erythema on the trunk and limbs and residual psoriatic plaques on the elbows at week 30. 


\begin{tabular}{|c|c|c|c|c|c|c|c|c|c|}
\hline 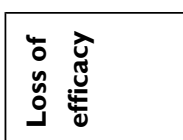 & $\stackrel{\circ}{z}$ & $\stackrel{0}{2}$ & $\stackrel{0}{\pi}$ & $\mathscr{0}$ & $\stackrel{0}{0}$ & $\stackrel{0}{0}$ & $\stackrel{0}{0}$ & $\stackrel{0}{0}$ & $\stackrel{\mathscr{0}}{*}$ \\
\hline 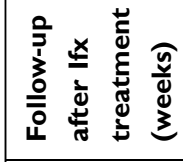 & $\frac{s}{2}$ & $\frac{\pi}{z}$ & $\frac{s}{z}$ & $\ddagger$ & $\frac{s}{z}$ & $\frac{s}{z}$ & $\frac{s}{z}$ & $\underline{\bar{g}}$ & $\Phi$ \\
\hline 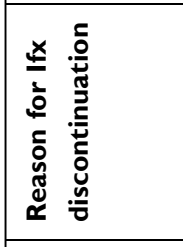 & 岁 & $\frac{\pi}{z}$ & 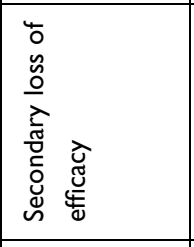 & 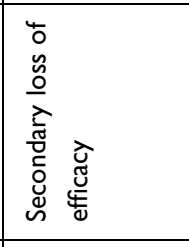 & 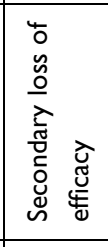 & 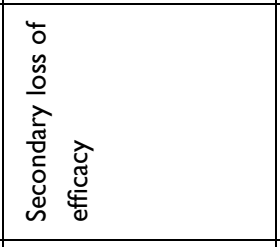 & 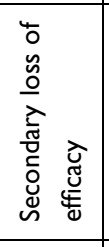 & 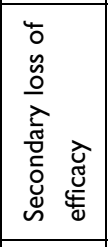 & 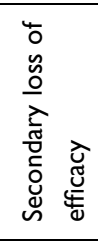 \\
\hline 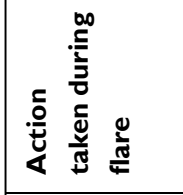 & $\frac{s}{z}$ & 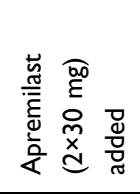 & 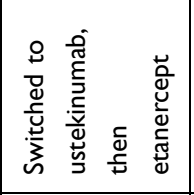 & 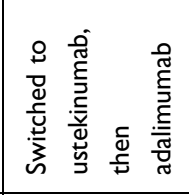 & 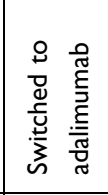 & 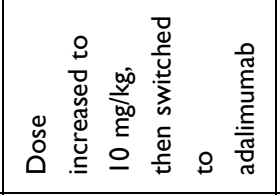 & $\frac{\pi}{z}$ & 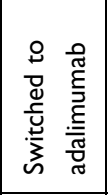 & 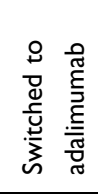 \\
\hline 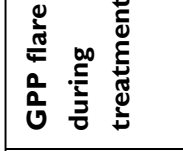 & \& & $\stackrel{\mathscr{0}}{.}$ & $\stackrel{\bullet}{\circ}$ & $\stackrel{\circ}{*}$ & 象 & $\stackrel{.0}{*}$ & $\stackrel{8}{z}$ & 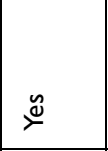 & $\stackrel{.0 .0}{*}$ \\
\hline 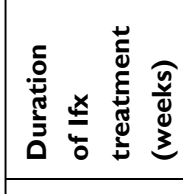 & 拉 & 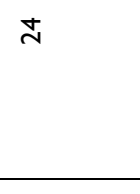 & 2 & 2 & $\mathscr{m}$ & $\cong$ & $\cong$ & 0 & $\pi$ \\
\hline 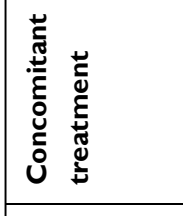 & z & 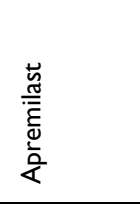 & $\stackrel{\circ}{z}$ & $\stackrel{\circ}{z}$ & z & $\stackrel{\circ}{z}$ & 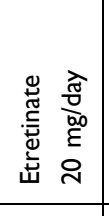 & $\stackrel{0}{z}$ & $\stackrel{\circ}{z}$ \\
\hline 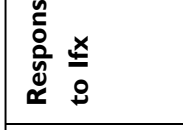 & $\stackrel{0}{\square}$ & 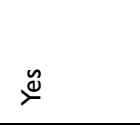 & 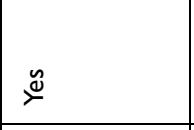 & $\stackrel{\mathscr{0}}{ٍ}$ & $\stackrel{\circ}{-}$ & $\stackrel{\circ}{-0}$ & $\stackrel{\mathscr{0}}{\beth}$ & 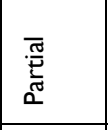 & $\stackrel{\mathscr{0}}{\longrightarrow}$ \\
\hline \begin{tabular}{|l}
$\stackrel{0}{0}$ \\
$\underline{o}$ \\
$\underline{\underline{x}}$ \\
\end{tabular} & $\frac{s}{z}$ & 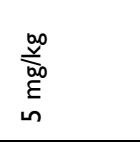 & $\begin{array}{l}\frac{g}{5} \\
\text { on } \\
2 \\
\end{array}$ & 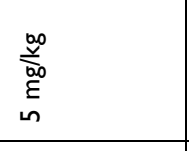 & 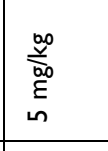 & 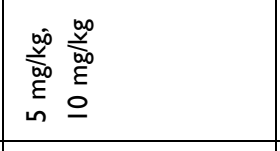 & 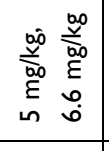 & 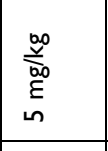 & 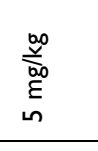 \\
\hline 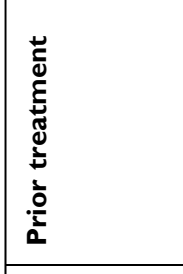 & $\begin{array}{l}0 \\
0 \\
0\end{array}$ & 岕 & 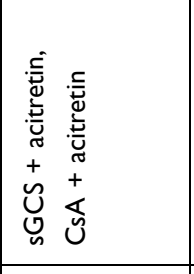 & 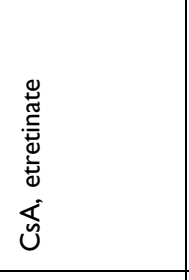 & \begin{tabular}{|l}
$\frac{0}{5}$ \\
$\frac{5}{2}$
\end{tabular} & 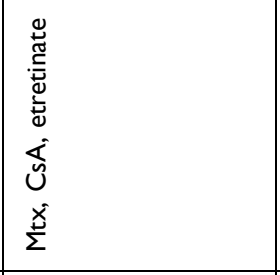 & 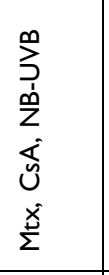 & 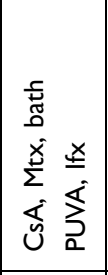 & $\begin{array}{l}\frac{x}{2} \\
\vdots \\
\vdots \\
0 \\
\end{array}$ \\
\hline 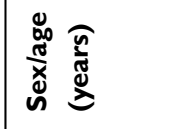 & 赵 & $\frac{o}{\Sigma}$ & $\overbrace{\tilde{\Sigma}}^{\infty}$ & 욘 & 㗭 & $\frac{n^{\circ}}{2}$ & $\frac{\gamma}{\Sigma}$ & $\frac{f}{\frac{F}{2}}$ & 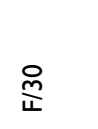 \\
\hline & 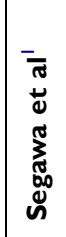 & 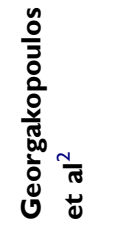 & 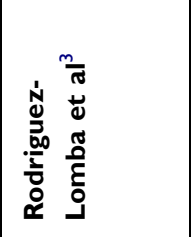 & 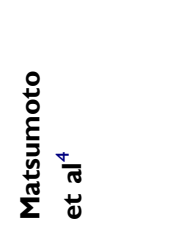 & & & & 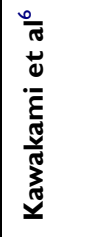 & \\
\hline
\end{tabular}




\begin{tabular}{|c|c|c|c|c|c|c|c|c|}
\hline 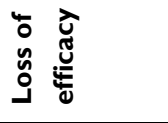 & 우 & \& & 2o & ż & $\stackrel{0}{z}$ & 우 & $\S$ & 우 \\
\hline 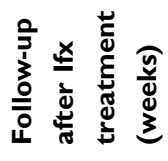 & $\mathbb{z}$ & $\S$ & $\S$ & $\S$ & $\S$ & $\hat{o}$ & 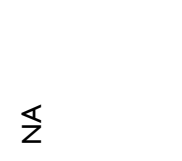 & 2 \\
\hline 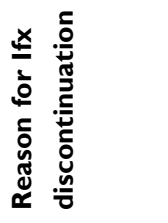 & $\S$ & $\S$ & $\S$ & $\S$ & 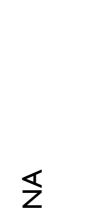 & 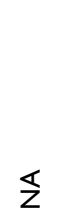 & 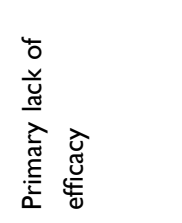 & $\S$ \\
\hline 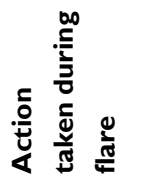 & $\mathbb{z}$ & $\overleftarrow{z}$ & $\S$ & $\S$ & $\overleftarrow{z}$ & $\S$ & 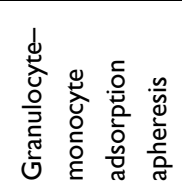 & 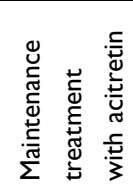 \\
\hline 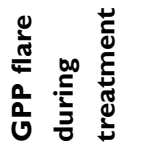 & 우 & $\stackrel{\circ}{z}$ & 우 & 우 & 2 & $\stackrel{\circ}{z}$ & $\stackrel{\mathscr{y}}{\nu}^{\mathscr{y}}$ & zo \\
\hline 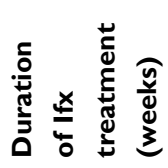 & $\underset{ \pm}{ \pm}$ & $\underset{+}{\ddagger}$ & 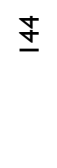 & $\stackrel{\infty}{+}$ & $\begin{array}{l}0 \\
0 \\
0 \\
0 \\
\frac{0}{00} \\
i=5\end{array}$ & 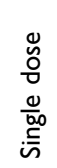 & $\S$ & $N$ \\
\hline 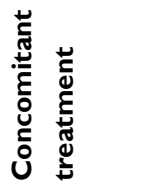 & $\stackrel{\circ}{z}$ & $\stackrel{\circ}{z}$ & $\stackrel{\circ}{z}$ & $\stackrel{\circ}{z}$ & 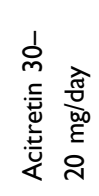 & $\sum^{x}$ & $\stackrel{\circ}{z}$ & 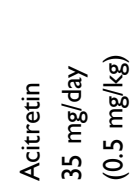 \\
\hline 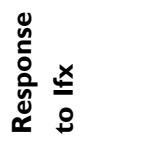 & $\underbrace{y}$ & $\stackrel{\tilde{y}}{\tau}$ & $\stackrel{\tilde{\nu}}{\check{\nu}}$ & $\stackrel{y}{\nu}$ & $\stackrel{\tilde{y}}{\nu}$ & $\stackrel{\mathscr{\nu}}{\tau}$ & $\stackrel{\circ}{z}$ & $\check{\nu}^{\underline{y}}$ \\
\hline 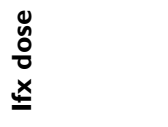 & 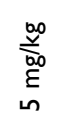 & 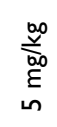 & 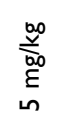 & $\begin{array}{c}\stackrel{\infty}{\stackrel{\infty}{0.0}} \\
\stackrel{5}{m}\end{array}$ & 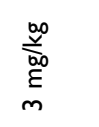 & 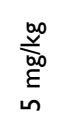 & $\begin{array}{l}\stackrel{\infty}{\stackrel{\infty}{0.0}} \\
\stackrel{5}{E} \\
n\end{array}$ & 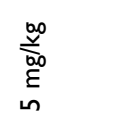 \\
\hline 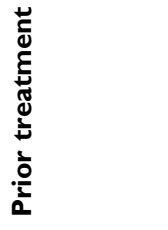 & 苑 & 苑 & $\overleftarrow{u}$ & 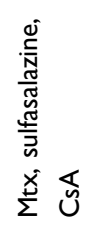 & $\begin{array}{l}0 \\
\text { Oे } \\
z\end{array}$ & $\frac{x}{\Sigma}$ & $\overleftarrow{u}$ & $\begin{array}{l}0 \\
\text { ô } \\
z\end{array}$ \\
\hline 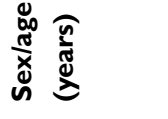 & 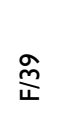 & $\frac{\text { సे }}{\Sigma}$ & $\begin{array}{l}\stackrel{L}{0} \\
\stackrel{0}{u}\end{array}$ & $\underset{\sqcup}{\bar{\Psi}}$ & $\stackrel{a}{\stackrel{2}{4}}$ & $\stackrel{m}{\Sigma}$ & $\frac{\mathfrak{F}}{4}$ & $\frac{N}{\Sigma}$ \\
\hline & 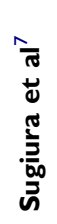 & & & 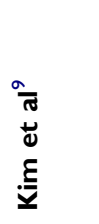 & & 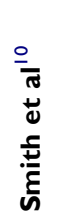 & 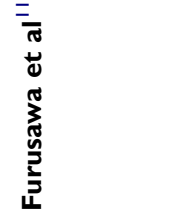 & 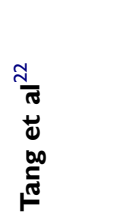 \\
\hline
\end{tabular}




\begin{tabular}{|c|c|c|c|c|c|c|c|c|c|c|c|c|}
\hline 울 & $\stackrel{0}{z}$ & $\stackrel{\circ}{z}$ & $\stackrel{0}{z}$ & $\S$ & $\stackrel{\circ}{z}$ & $\stackrel{\tilde{u}}{\tau}$ & \& & z & z & 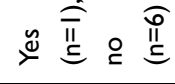 & $\stackrel{0}{z}$ & $\stackrel{\circ}{z}$ \\
\hline$\S$ & $\S$ & 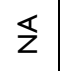 & $\S$ & $\S$ & $\S$ & $\S$ & $\S$ & $\S$ & $\S$ & $\S$ & $\stackrel{\infty}{\sim}$ & 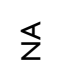 \\
\hline 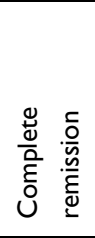 & 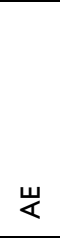 & 屵 & 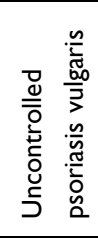 & 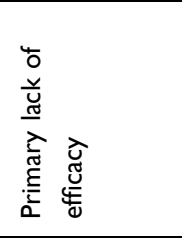 & 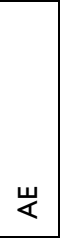 & 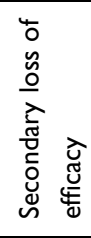 & 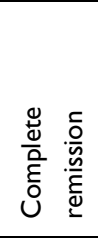 & ए & 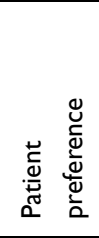 & 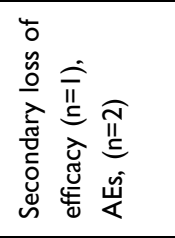 & 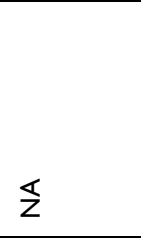 & $\S$ \\
\hline$\S$ & $\S$ & $\S$ & 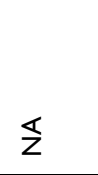 & 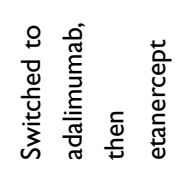 & 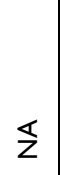 & 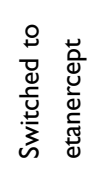 & $\mathbb{z}$ & 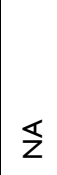 & 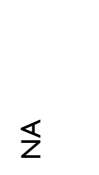 & $\mathbb{z}$ & 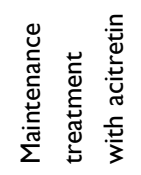 & $\S$ \\
\hline$\S$ & $\S$ & $\stackrel{0}{z}$ & i & $\S$ & $\S$ & $\stackrel{\mathscr{y}}{\tau}$ & $\S$ & 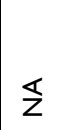 & $\S$ & 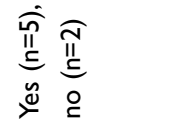 & 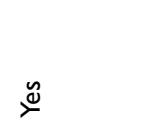 & zo \\
\hline $\begin{array}{l}\mathscr{\nu} \\
\stackrel{0}{0} \\
\frac{0}{60} \\
\stackrel{\infty}{\infty}\end{array}$ & $\stackrel{\infty}{+}$ & $\infty$ & $\stackrel{\sim}{\sim}$ & $\simeq$ & $N$ & $\bar{N}$ & 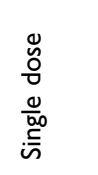 & 0 & 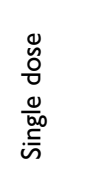 & 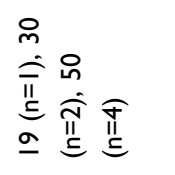 & $\begin{array}{l}0 \\
00 \\
0 \\
\frac{0}{00} \\
\stackrel{0}{=}\end{array}$ & $\approx$ \\
\hline$\S$ & $\mathbb{z}$ & $\S$ & $\S$ & $\overleftarrow{z}$ & $\mathbb{z}$ & 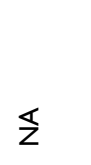 & $\S$ & $\S$ & 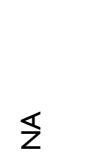 & $\begin{array}{l}\underset{\pi}{\pi} \\
\stackrel{0}{\tilde{y}} \\
0\end{array}$ & 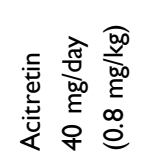 & $\stackrel{\circ}{z}$ \\
\hline$\stackrel{\mathscr{y}}{\check{y}}$ & $\stackrel{\mathscr{y}}{\check{\nu}}$ & $\stackrel{\mathscr{y}}{\check{\nu}}$ & $\stackrel{y}{\check{\nu}}$ & ㅇ & $\stackrel{\Xi}{\check{E}}$ & $\stackrel{\mathscr{y}}{\check{\nu}}$ & $\stackrel{\check{\Delta}}{\nu}$ & $\stackrel{\check{s}}{\nu}$ & $\stackrel{\tilde{y}}{\nu}$ & $\stackrel{\mathscr{\Xi}}{\nu}$ & $\stackrel{\check{y}}{\nu}$ & $\stackrel{\check{g}}{\nu}$ \\
\hline$\S$ & $\S$ & $\S$ & $\S$ & $\S$ & $\S$ & $\mathbb{z}$ & $\S$ & $\Sigma$ & 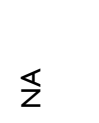 & & $\underset{n}{\stackrel{\infty}{\stackrel{\infty}{0}_{0}^{0}}}$ & 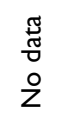 \\
\hline$\varangle$ & $\varangle$ & 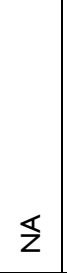 & $\S$ & 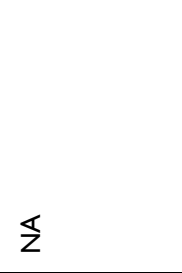 & $\varangle$ & 乙 & 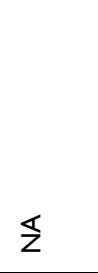 & $\S$ & 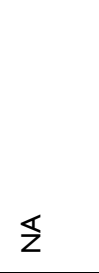 & 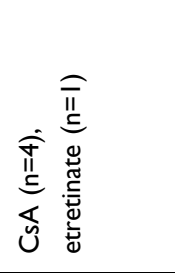 & 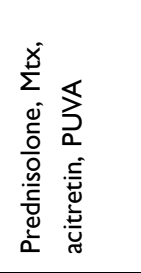 & 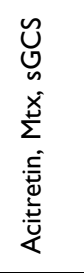 \\
\hline$\underset{\Downarrow}{\overleftarrow{4}}$ & $\frac{\nwarrow}{\Sigma}$ & $\underset{\longleftrightarrow}{\overleftarrow{\Psi}}$ & $\frac{\overleftarrow{z}}{\Sigma}$ & $\underset{\text { \} }{\overleftarrow{4}}} &{\underset{\mathbf{Z}}{\mathbb{Z}}} &{\underset{\text { z }}{\mathbb{Z}}} &{\underset{\mathbf{z}}{\overleftarrow{u}}} &{\underset{\mathbf{L}}{\mathbb{Z}}} &{\underset{\longleftarrow}{\overleftarrow{\psi}}} &{\text { 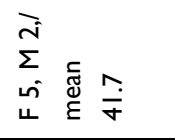 }} &{\text { 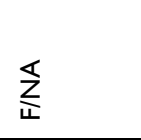 }} &{\frac{\overleftarrow{z}}{\Sigma}} \\
{\hline \text { 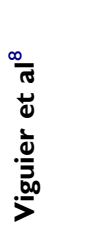 }} &{ } &{ } &{ } &{ } &{ } &{ } &{ } &{ } &{ } &{\text { 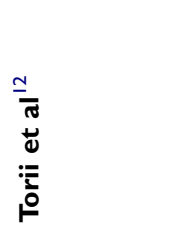 }} &{\text { 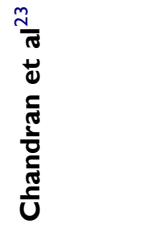 }} &{\text { 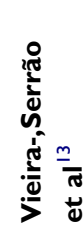 }} \\
$\hline
\end{tabular}




\begin{tabular}{|c|c|c|c|c|c|c|c|c|c|c|c|}
\hline 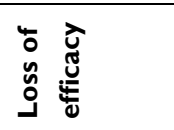 & $\stackrel{0}{z}$ & $\stackrel{0}{z}$ & zo & $\stackrel{0}{z}$ & 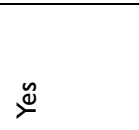 & $\stackrel{0}{z}$ & $\stackrel{0}{z}$ & $\stackrel{0}{z}$ & $\stackrel{0}{z}$ & $\stackrel{\circ}{z}$ & $\stackrel{0}{z}$ \\
\hline 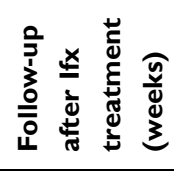 & 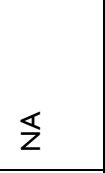 & 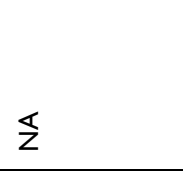 & $\stackrel{\Delta}{\Lambda}$ & $\stackrel{ \pm}{ \pm}$ & $\underline{z}$ & $\simeq$ & $\underline{z}$ & $\frac{\pi}{z}$ & 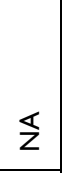 & $\simeq$ & + \\
\hline 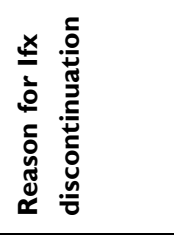 & 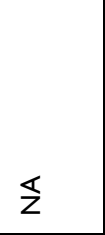 & 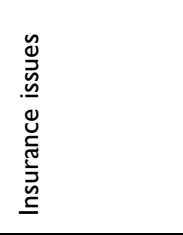 & 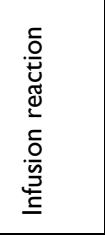 & $\underline{z}$ & $\underline{z}$ & $\stackrel{\Sigma}{z}$ & $\mathbb{z}$ & $\mathbb{z}$ & $\mathbb{z}$ & $\stackrel{\pi}{z}$ & $\Sigma$ \\
\hline 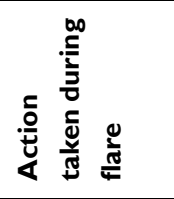 & 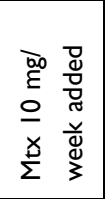 & 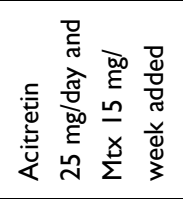 & 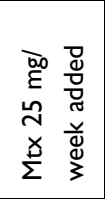 & 可 & 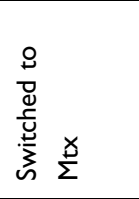 & 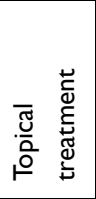 & $\mathbb{Z}$ & $\mathbb{z}$ & $\mathbb{z}$ & 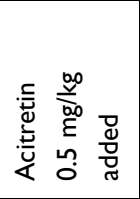 & 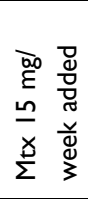 \\
\hline 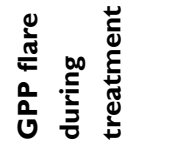 & $\stackrel{\check{\nu}}{\tau}$ & $\stackrel{\check{\varpi}}{\check{\nu}}$ & $\stackrel{\check{\nu}}{\tau}$ & $\stackrel{\mathscr{\Xi}}{0}$ & $\stackrel{\check{g}}{\check{\nu}}$ & $\stackrel{0}{\check{0}}$ & $\stackrel{0}{z}$ & $\stackrel{2}{z}$ & $\stackrel{0}{z}$ & $\stackrel{\mathscr{y}}{\check{0}}$ & $\stackrel{\square}{\check{\Sigma}}$ \\
\hline 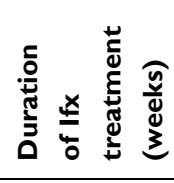 & \pm & 8 & $\simeq$ & 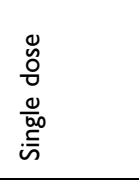 & 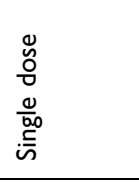 & $\simeq$ & $\frac{t}{\Lambda}$ & $\frac{t}{\Lambda}$ & 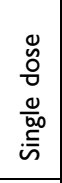 & $\bar{\lambda}$ & ${ }^{\circ}$ \\
\hline 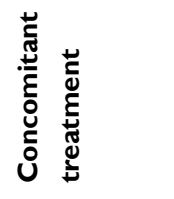 & $\frac{x}{\Sigma}$ & 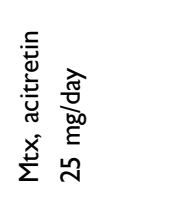 & $\frac{x}{\Sigma}$ & $\begin{array}{l}\frac{0}{0} \\
\frac{0}{0} \\
\frac{0}{\bar{E}} \\
\frac{0}{0} \\
\frac{0}{2}\end{array}$ & zo & $\stackrel{\circ}{z}$ & zo & 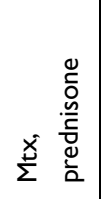 & $\stackrel{\circ}{z}$ & 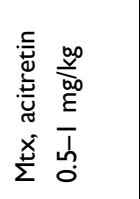 & $\frac{x}{\Sigma}$ \\
\hline 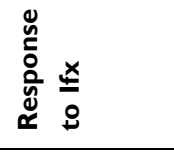 & $\stackrel{\mathscr{s}}{\check{0}}$ & 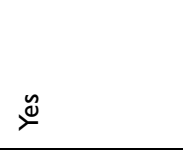 & $\stackrel{\mathscr{E}}{\check{\nu}}$ & 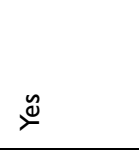 & $\stackrel{\check{\Xi}}{\check{\Sigma}}$ & 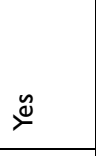 & $\overbrace{}^{\check{\Xi}}$ & 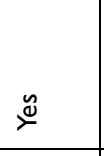 & $\stackrel{\check{\Xi}}{\check{\Sigma}}$ & 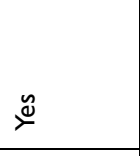 & 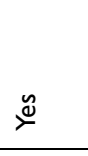 \\
\hline 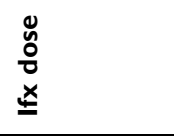 & 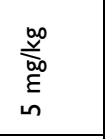 & 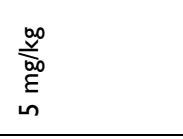 & 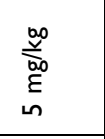 & 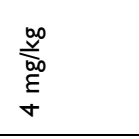 & 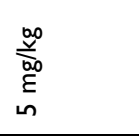 & 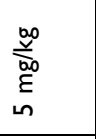 & 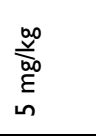 & 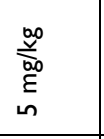 & 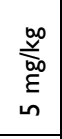 & 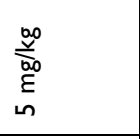 & 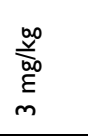 \\
\hline 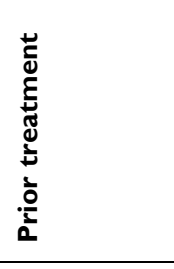 & 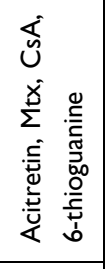 & 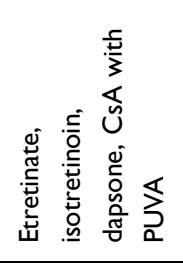 & 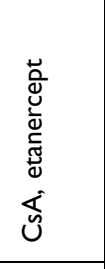 & 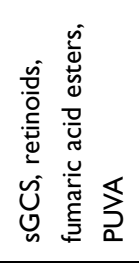 & $\begin{array}{l}\text { 号 } \\
\text { ż }\end{array}$ & 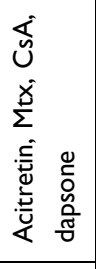 & 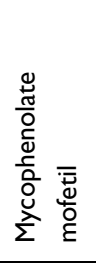 & 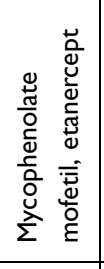 & 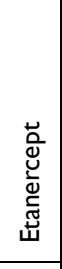 & 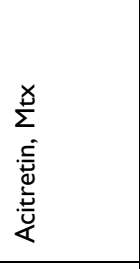 & 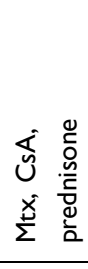 \\
\hline 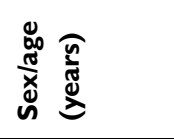 & 点 & 点 & $\frac{3}{4}$ & 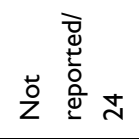 & 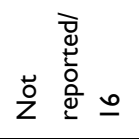 & 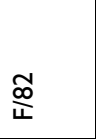 & $\hat{\bar{u}}$ & $\frac{f}{4}$ & $\tilde{\tilde{n}}$ & $\frac{\bar{\rho}}{\Sigma}$ & 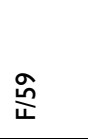 \\
\hline & 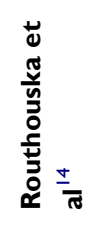 & & & 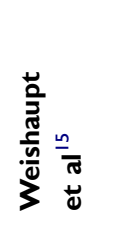 & & 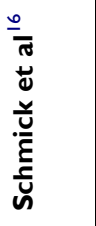 & 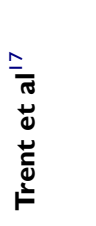 & & & 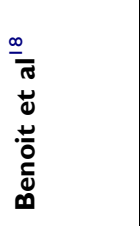 & 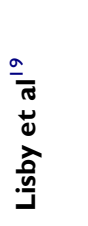 \\
\hline
\end{tabular}




\begin{tabular}{|c|c|c|c|}
\hline 으 & 우 & 우 & zo \\
\hline$\stackrel{m}{0}$ & $\frac{0}{\wedge}$ & $\S$ & $\S$ \\
\hline$\S$ & $\S$ & $\begin{array}{l}\stackrel{\infty}{\circ} \\
\stackrel{\overline{0}}{0} \\
\text { o. } \\
0 \\
\underline{x}\end{array}$ & 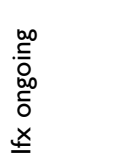 \\
\hline$\S$ & $\S$ & 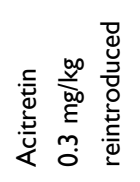 & 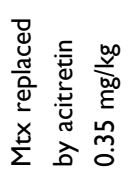 \\
\hline 乏̌ & zo & $\stackrel{\varpi}{\check{\varpi}}$ & $\check{\check{\nu}}$ \\
\hline 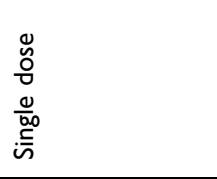 & 0 & 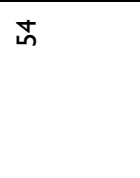 & in \\
\hline ㅇ & 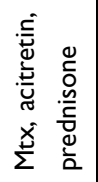 & 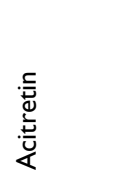 & $\stackrel{x}{\Sigma}$ \\
\hline$\stackrel{\mathscr{\Xi}}{\succ}$ & $\stackrel{\mathscr{\Xi}}{\succ}$ & $\stackrel{\Xi}{\check{\Xi}}$ & $\stackrel{\Xi}{\check{\nu}}$ \\
\hline 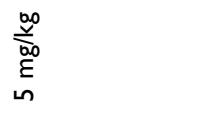 & 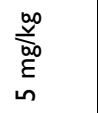 & $\begin{array}{l}\stackrel{\infty}{\stackrel{\infty}{00}} \\
\sum_{n}^{\infty}\end{array}$ & 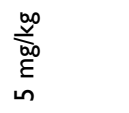 \\
\hline 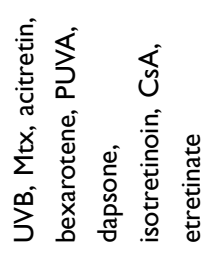 & 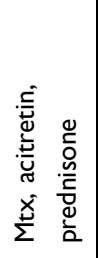 & 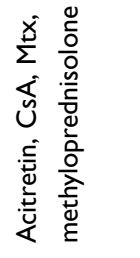 & $\begin{array}{l}\stackrel{0}{\tilde{\nu}} \\
\text { z }\end{array}$ \\
\hline$\frac{+}{4}$ & $\frac{a}{\tilde{\Sigma}}$ & $\frac{n}{\underline{p}}$ & $\frac{\circ}{\Sigma}$ \\
\hline 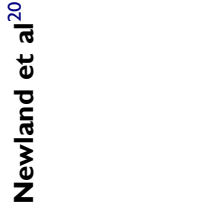 & 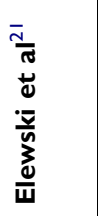 & 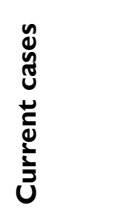 & \\
\hline
\end{tabular}

was most commonly utilized. ${ }^{6,7,9,13,17,19,21}$ However, irregular dosing, only in the event of pustular flares, was also used in some cases. ${ }^{14}$ Despite the fact that Ifx induces rapid resolution of pustules within 1-8 days, monotherapy was frequently insufficient to provide long-term disease control. Pustular flares during Ifx treatment ${ }^{2-4,6,8,11,12,14,16,18,19,23}$ and secondary loss of efficacy ${ }^{3,4,6,8,12}$ were observed in a majority of reports. Various strategies were undertaken in such cases, including the addition of $\mathrm{Mtx}^{14,15,19}$ or acitretin $^{14,18,22,23}$ or switching to adalimumab, ${ }^{4,6,8}$ etanercept, ${ }^{3,8}$ or ustekinumab. ${ }^{3,4}$ Ifx survival calculated on the basis of reports available in the literature and current cases indicates that nearly $70 \%$ of patients with GPP are treated with Ifx for at least 1 year and $40 \%$ of patients for about 3 years (Figure 3).

Ifx is commonly used in combination with Mtx not only to improve therapeutic response but also to avoid formation of anti-Ifx neutralizing antibodies. ${ }^{14,17-19,21}$ Combined use of Ifx and acitretin is less documented in the literature. ${ }^{9,14,18,22,23}$ In one report of severe GPP in a patient with neutrophilic cholangitis, acitretin $0.8 \mathrm{mg} / \mathrm{kg}$ was introduced as concurrent systemic treatment on day 6 after a single dose of Ifx, and the patient remained pustule-free during a 7-month follow-up. ${ }^{23}$ In another report, Tang et $\mathrm{al}^{22}$ administered Ifx in combination with acitretin $(0.5 \mathrm{mg} / \mathrm{kg})$, which resulted in rapid amelioration of GPP within 48 hours. In total, two infusions of Ifx were given and acitretin was tapered down to $10 \mathrm{mg} /$ day, which enabled satisfactory control of the disease over a 24-month follow-up. In our patients, the combination of Ifx and acitretin (0.3$0.35 \mathrm{mg} / \mathrm{kg}$ ) was associated with good therapeutic effects. In addition, the therapy was well tolerated and no adverse events, usually common with doses above $0.5 \mathrm{mg} / \mathrm{kg}$, were noted.

\section{Conclusion}

Ifx is a safe and highly effective treatment option for GPP. Rapid amelioration of the condition may be observed after a single Ifx dose, which makes Ifx a suitable option for "rescue treatment" in the most severe cases. Ifx may be also safely combined with lowdose acitretin in the treatment of GPP to achieve longterm control of the disease. Nevertheless, larger randomized studies are needed to compare the efficacy of monotherapy with Ifx versus combination therapy of Ifx with low-dose acitretin in GPP. 


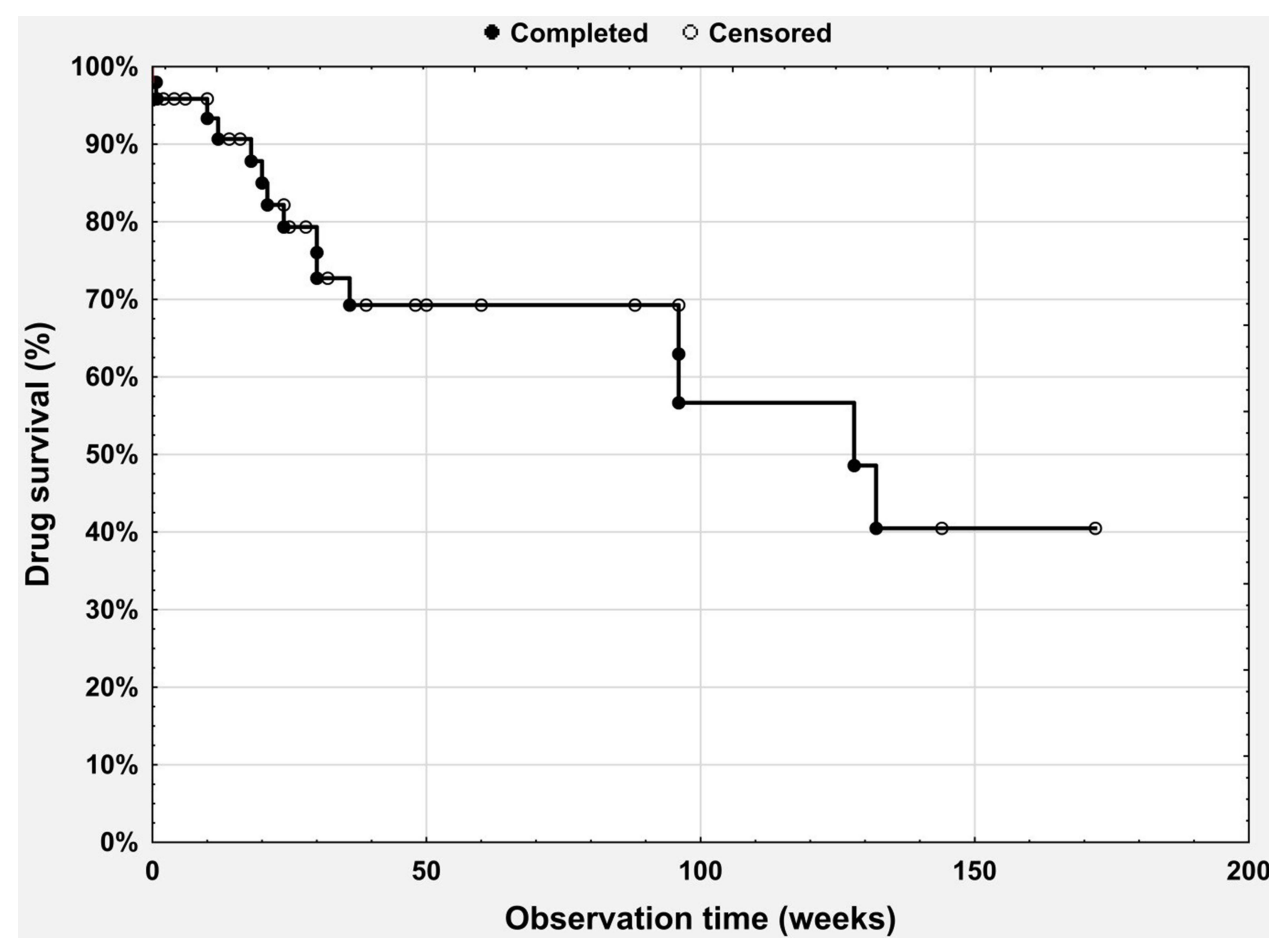

Figure 3 Infliximab (Ifx) survival in GPP calculated on the basis of cases available in the literature.

\section{Disclosure}

Marta Kołt-Kamińska and Magdalena Żychowska declare no conflicts of interest for this work. Adam Reich reports personal fees from AbbVie, Novartis, Chema Rzeszów, Galderma, Pfizer, Eli Lilly, Trevi Therapeutics, Dermira, Janssen, BMS, and GlaxoSmithKline outside the submitted work, and has been a consultant or speaker for AbbVie, Bioderma, Celgene, Chema Elektromet, Eli Lilly, Galderma, Janssen, Leo Pharma, Medac, Menlo Therapeutics, Novartis, PierreFabre, Sandoz, and Trevi and principal investigator or subinvestigator in clinical trials sponsored by AbbVie, Drug Delivery Solutions, Galderma, Genentech, Janssen, Kymab Limited, Leo Pharma, Menlo Therapeutics, MetrioPharm, MSD, Novartis, Pfizer, and Trevi. He reports no other potential conflicts of interest for this work.

\section{References}

1. Segawa Y, Ishida R, Kanehisa F, et al. IgA nephropathy in a patient receiving infliximab for generalized pustular psoriasis. BMC Nephrol. 2020;21:366. doi:10.1186/s12882-020-02015-0

2. Georgakopoulos JR, Ighani A, Yeung J. Short- and long-term management of an acute pustular psoriasis flare: a case report. J Cutan Med Surg. 2017;21:452-456. doi:10.1177/1203475417712499

3. Rodríguez-Lomba E, Baniandrés $\mathrm{O}$, Cano N, Suárez-Fernández R. Generalized pustular psoriasis and neutrophilic cholangitis: an infrequently reported association with excellent response to tumour necrosis factor inhibitors. Australas J Dermatol. 2017;58:70-71. doi:10. 1111/ajd.12471
4. Matsumoto A, Komine M, Karakawa M, Kishimoto M, Ohtsuki M. Adalimumab administration after infliximab therapy is a successful treatment strategy for generalized pustular psoriasis. $J$ Dermatol. 2017;44:202-204. doi:10.1111/1346-8138.13632

5. Robinson A, Van Voorhees AS, Hsu S, et al. Treatment of pustular psoriasis: from the Medical Board of the National Psoriasis Foundation. J Am Acad Dermatol. 2012;67(2):279-288. doi:10. 1016/j.jaad.2011.01.032

6. Kawakami H, Maeda T, Abe N, et al. Efficacy of adalimumab and methotrexate combination therapy on generalized pustular psoriasis patients unresponsive to infliximab monotherapy due to anti-infliximab antibody development. $J$ Dermatol. 2015;42:94-95. doi:10.1111/1346-8138.12704

7. Sugiura K, Endo K, Akasaka T, Akiyama M. Successful treatment with infliximab of sibling cases with generalized pustular psoriasis caused by deficiency of interleukin-36 receptor antagonist. $J$ Eur Acad Dermatol Venereol. 2015;29:2054-2056. doi:10.1111/ jdv. 12590

8. Viguier M, Aubin F, Delaporte E, et al. Efficacy and safety of tumor necrosis factor inhibitors in acute generalized pustular psoriasis. Arch Dermatol. 2012;148:1423-1425. doi:10.1001/2013.jamadermatol.80

9. Kim HS, You HS, Cho HH, et al. Two cases of generalized pustular psoriasis: successful treatment with infliximab. Ann Dermatol. 2014;26:787-788. doi:10.5021/ad.2014.26.6.787

10. Smith N, Harms KL, Hines AC, et al. Acute treatment of generalized pustular psoriasis of von Zumbusch with single-dose infliximab. $J \mathrm{Am}$ Acad Dermatol. 2013;68:e187-9. doi:10.1016/j.jaad.2012.11.017

11. Furusawa K, Hasegawa T, Ikeda S. Immunosuppressant and infliximab-resistant generalized pustular psoriasis successfully treated with granulocyte and monocyte adsorption apheresis. Ther Apher Dial. 2012;16:379-380. doi:10.1111/j.1744-9987.2012.01067.x

12. Torii $\mathrm{H}$, Nakagawa $\mathrm{H}$; Japanese Infliximab Study Investigators. Long-term study of infliximab in Japanese patients with plaque psoriasis, psoriatic arthritis, pustular psoriasis and psoriatic erythroderma. J Dermatol. 2011;38:321-334. doi:10.1111/j.13468138.2010.00971.x 
13. Vieira-Serrão V, Martins A, Paiva Lopes MJ. Infliximab in recalcitrant generalized pustular arthropathic psoriasis. Eur J Dermatol. 2008;18:71-73.

14. Routhouska SB, Sheth PB, Korman NJ. Long-term management of generalized pustular psoriasis with infliximab: case series. J Cutan Med Surg. 2008;12:184-188. doi:10.2310/7750.2008.07036

15. Weishaupt C, Metze D, Luger TA, Ständer S. Treatment of pustular psoriasis with infliximab. J Dtsch Dermatol Ges. 2007;5:397-399. doi:10.1111/j.1610-0387.2007.06296.x

16. Schmick K, Grabbe J. Recalcitrant, generalized pustular psoriasis: rapid and lasting therapeutic response to antitumour necrosis factor-alpha antibody (infliximab). Br J Dermatol. 2004;150(2):367. doi:10.1111/j.1365-2133.2004.05719.x

17. Trent JT, Kerdel FA. Successful treatment of Von Zumbusch pustular psoriasis with infliximab. J Cutan Med Surg. 2004;8:224-228. doi:10.1177/120347540400800404

18. Benoit S, Toksoy A, Bröcker EB, Gillitzer R, Goebeler M. Treatment of recalcitrant pustular psoriasis with infliximab: effective reduction of chemokine expression. Br J Dermatol. 2004;150:1009-1012. doi:10.1111/j.1365-2133.2004.05960.x
19. Lisby S, Gniadecki R. Infliximab (Remicade) for acute, severe pustular and erythrodermic psoriasis. Acta Derm Venereol. 2004;84: 247-248.

20. Newland MR, Wienstein A, Kerdel F. Rapid response to infliximab in severe pustular psoriasis, von Zumbusch type. Int J Dermatol. 2002;41:449-452. doi:10.1046/j.1365-4362.2002.01543.x

21. Elewski BE. Infliximab for the treatment of severe pustular psoriasis. J Am Acad Dermatol. 2002;47:796-797. doi:10.1067/mjd.2002. 128382

22. Tang MM, Spanou Z, Tang $\mathrm{H}$, et al. Rapid downregulation of innate immune cells, interleukin-12 and interleukin-23 in generalized pustular psoriasis with infliximab in combination with acitretin. Dermatology. 2012;225(4):338-343. doi:10.1159/00034 6243

23. Chandran NS, Chong WS. A dramatic response to a single dose of infliximab as rescue therapy in acute generalized pustular psoriasis of von Zumbusch with a neutrophilic cholangitis. Australas J Dermatol. 2010;51(1):29-31. doi:10.1111/j.1440-0960.2009.00 588.x

\section{Publish your work in this journal}

Biologics: Targets and Therapy is an international, peer-reviewed journal focusing on the patho-physiological rationale for and clinical application of Biologic agents in the management of autoimmune diseases, cancers or other pathologies where a molecular target can be identified. This journal is indexed on PubMed Central, CAS, EMBase,
Scopus and the Elsevier Bibliographic databases. The manuscript management system is completely online and includes a very quick and fair peer-review system, which is all easy to use. Visit http://www.dovepress.com/testimonials.php to read real quotes from published authors. 